\title{
Filles déscolarisées et apprentissage des « métiers masculins » dans la ville de Kinshasa : quelle image sociale?
}

\author{
Dieudonné Musa Alokpo \\ Département des Sciences de l'Education, Université de Kinshasa, \\ République Démocratique du Congo
}

\begin{abstract}
In the city of Kinshasa there has been a marked increase in the numbers of women expressing an interest in jobs traditionally thought of as male occupations. The case of the out-of-school girls learning mechanics is a good illustration of this new phenomenon. In a society in which access to employment in the formal sector is saturated, apprenticeships organized in non-formal educational centers offer out-of-school girls the opportunity to develop practical skills that can be used immediately, and so help them create their own employment opportunities. The choice to learn the 'male occupation' of mechanics contrasts with the cultural conceptions about the boundaries between the female and male oriented work. Considering such a contrast, and the recurring political discourse concerning gender equality, which encourages women to integrate into all spheres of social activity, it is interesting to question the inhabitants of Kinshasa about the relevance of the occupation choices of the out-of-school girls and about their skill levels in the "male jobs" arena. The survey showed that the inhabitants of Kinshasa are against the division of work along gender lines and remain in favor of the idea that men and women should be able to pursue jobs according to their skills rather than their sex.
\end{abstract}

Key words: male jobs, apprenticeships, social representation

L'intérêt porté par les femmes à l'exercice des métiers initialement reconnus aux hommes, est un fait nouveau en émergence dans la ville de Kinshasa. Le cas des filles déscolarisées apprenant la mécanique en est une illustration éloquente. Dans une société en crise où l'accès à l'emploi formel est saturé, les apprentissages organisés dans les centres d'éducation non formelle offrent aux filles déscolarisées l'opportunité de développer des compétences pratiques immédiatement utilisables, de se créer l'emploi et de gagner leur vie. Ce choix d'apprendre la mécanique, métier dit "masculin », contraste cependant avec les conceptions culturelles établissant la démarcation entre les travaux féminins et masculins. Considérant un tel contraste, et l'amplification du discours sur la parité et l'égalité de sexe, lequel encourage les femmes à s'engager dans toutes les sphères d'activités, il est intéressant d'interroger les habitants de Kinshasa sur la pertinence du choix professionnel des filles déscolarisées et sur leur niveau des compétences dans les « métiers masculins ». Les données de l'enquête indiquent que les habitants de Kinshasa sont opposés à la division sexuelle du travail et demeurent plutôt favorables à l'exercice indistinct des métiers, selon les compétences de chaque individu, homme ou femme.

Mots clés : métiers masculins, déscolarisation, représentation sociale 


\section{Introduction}

L'accès des populations à l'éducation et à l'emploi, ainsi que les rapports de genre, sont étroitement liés au processus historique et culturel de chaque peuple ; plus particulièrement, à la capacité de chaque Etat à négocier les changements intervenus dans l'environnement international. En effet, les politiques néolibérales ont entraîné la recomposition de l'économie mondiale, affectant notablement les économies nationales, spécialement celles des pays du Sud, avec des conséquences considérables sur le fonctionnement du marché du travail et la structure de l'emploi.

A cet effet, Chataigner et Magro (2007: 524) allèguent que : "L'imposition aux Etats africains des normes internationales non adaptées à la dynamique historique et l'amoindrissement des responsabilités et des capacités de l'Etat dans le pays en transition, ont été les principales causes de la faillite de l'Etat. " Il s'en est suivi la réduction sensible du secteur formel, l'extension de l'informel et le renforcement des inégalités.

Plusieurs chercheurs tant du Nord que du Sud (Duruflé, I988; Massiah, 2000; Trefon, 2003) partagent cette analyse, tout en insistant sur des effets particuliers des programmes d'ajustement structurel, tels la baisse du pouvoir d'achat des ménages, l'accentuation des inégalités et de la précarité, lesquels ont aggravé la conjoncture de crise que traversent beaucoup des pays du sud, entraînant une forte détérioration des conditions sociales et les restrictions dans l'accès au marché du travail, notamment à l'égard des femmes.

En République démocratique du Congo, la période post-conflit ${ }^{\mathrm{I}}$ a établi quelques ouvertures susceptibles de changer la physionomie du marché du travail formel, par rapport à la question du genre. En effet, la constitution de la république (2006) et la loi sur la parité, en consacrant le principe de l'égalité entre les sexes en matière d'accès à l'emploi et aux avantages y afférents, ont permis de compter en République Démocratique du Congo, aujourd'hui plus que dans le passé, un nombre élevé de femmes assumant des hautes charges publiques ${ }^{2}$, bien que leur effectif soit encore inférieur à celui des hommes. Ces femmes ont eu la chance d'accéder à l'école et d'obtenir un diplôme dont elles se prévalent pour la recherche de l'emploi dans le secteur structuré. Ce qui n'est malheureusement pas le cas pour beaucoup d'autres qui n'ont eu cette opportunité.

Car en dépit des efforts déployés, l'Etat congolais n'est pas encore parvenu à assurer la scolarisation universelle aux enfants et aux jeunes, ni à créer des emplois convenables et à répondre aux besoins du travail. Cette condition a entraîné la déscolarisation de nombreux jeunes, dont un important effectif des filles. Celles-ci viennent grossir le rang d'autres filles exclues de l'école pour des raisons culturelles. A celles-là, s'ajoutent également des filles qui ont interrompu les études suite à des grossesses ou mariages précoces. Se trouvant dans un état où elles ne peuvent ni étudier ni travailler, ces jeunes filles sont exposées à toutes les pratiques dépravantes, tels vol, prostitution, mendicité,

I L'accord politique signé en 2003 à Sun City, entre le gouvernement congolais et les groupes armés, a institué une période de transition qui débouche sur les élections de novembre 2006.

2 L'allusion est ici faite aux femmes ministres, sénatrices, députées, administrateurs-délégués généraux des entreprises publiques, conseillères dans les cabinets politiques, etc... 
etc. Elles deviennent un véritable problème pour la société et constituent une perte de capital humain dans cette période de reconstruction du pays où il $\mathrm{y}$ a, plus que jamais, nécessité de prendre appui sur toutes les compétences, sans discrimination d'origine sociale, de religion ou de sexe. En contexte de récession, les filles déscolarisées ne tiennent leur salut qu'au secteur informel où les femmes jouent habituellement un rôle important pour la survie des ménages et sont largement engagées dans les activités de subsistance (maraîchage, petits commerces, tressage de cheveux, etc.), considérées comme "féminines".

\section{Crise congolaise et intérêt des filles pour les métiers dits masculins}

L'éducation et le travail étant, pour la personne humaine, des droits universels et inaliénables (Déclaration universelle des droits de l'homme, Art. 23 et Art. 26), une fille qui n'étudie pas se trouve privée d'un important moyen de développer ses connaissances et de partager la culture universelle. Elle vit dans l'ignorance de sa personne et de ses capacités. Car, « vivre sans un minimum d'éducation c'est vivre en sursis ». De même, celle qui ne travaille pas et qui n'a pas de revenu, s'appauvrit et appauvrit également son milieu.

En tant que valeur sociale et économique, le travail est le seul moyen d'assurer l'épanouissement humain, de créer la richesse et de transformer l'environnement.

Dans un contexte de faillite de l'Etat, l'atteinte de ces niveaux de réalisation demeure un leurre, tant que les déclarations de bonnes intentions ne seront pas assorties des politiques concrètes qui puissent permettre d'assurer aux populations le bien-être et l'accès aux services sociaux dont l'emploi. L'exemple de la ville de Kinshasa, évoqué par Trefon (2003: I6) est éloquent, lorsqu'il affirme qu' " avec la crise humanitaire grave issue de la faillite de l'Etat et de la mauvaise gouvernance, les Kinois, contrairement à la plupart des populations urbaines ne considèrent plus comme allant de soit, la santé, l'éducation, la sécurité alimentaire, le transport en commun et l'emploi".

Cette situation a favorisé l'accroissement des activités du secteur informel, où nombre d'initiatives d'éducation et de formation extrascolaires ont connu une grande expansion. Ces formations dites " non formelles " renferment, selon Simkins (I977: 35), "toute activité d'éducation organisée en dehors du système formel établi, orientée vers une clientèle bien identifiée avec des objectifs d'apprentissage bien définis." Elles offrent des programmes de courtes durées, allant de l'alphabétisation à l'apprentissage des métiers ; ce faisant, elles constituent des opportunités de récupération et d'encadrement des déscolarisées et autres désœuvrées.

Parmi les formations organisées, l'on dénombre : l'esthétique, la coiffure, la coupe et la couture, le dépannage des cellulaires, les technologies appropriés, le dépannage électronique et la mécanique. Les filles s'inscrivent indistinctement dans ces différents métiers où elles développent des compétences remarquables, à l'instar des garçons. Or, certains métiers comme la réparation des radios et la mécanique sont considérés, depuis toujours à Kinshasa, comme "masculins ". Ils sont cependant aujourd'hui embrassés par les femmes qui s'y prennent tout aussi bien. En effet, les barrières culturelles et les mauvaises conceptions sur le rôle de la femme et la limitation des métiers qu'elle devait 
exercer, ont constitué durant des décennies, des obstacles à son épanouissement. Ainsi que l'affirme Séhili (2003:35), " tout ce qui relève de la technique semble être socialement construit comme domaine masculin." Et, Berner (I997: 8) de renchérir : "Seuls les hommes sont sensés posséder des compétences techniques, et la maîtrise de la technologie est fortement identifiée à la masculinité».

Cette discrimination au détriment de la femme a dépossédé la société de sa contribution dans l'effort de production et dans la solution des problèmes spécifiques se posant dans son entourage.

L'enquête préliminaire menée auprès de quelques centres d'éducation non formelle à Kinshasa, particulièrement ceux organisant la mécanique et l'électronique, a abouti aux résultats qui constituent un vibrant démenti à cette conception qui excluait la femme de certaines professions, préjugeant de son incapacité à les exercer. Il en ressort une présence accrue des filles dans les différents garages et ateliers électroniques, apprenant les métiers dits " masculins ". Cette réalité requiert un changement de mentalité favorable à la déconstruction des conceptions discriminatoires entre homme et femme, lesquelles ne se justifient ni au plan du droit ni à celui de la compétence.

De là, apparait l'intérêt d'évaluer l'attitude de la population de Kinshasa face au choix professionnel des filles déscolarisées (c'est-à-dire les différentes représentations relatives à la femme exerçant les métiers dits " masculins "). Aussi, de prolonger la réflexion pour découvrir les mobiles de ce choix professionnel. Car, le besoin de survie dans un contexte de pauvreté et de faillite de l'Etat, évoqué ci-haut, ne nous semble pas être les seuls déterminants du choix spécifique des «métiers masculins ». Enfin, d'observer et d'obtenir l'appréciation des employeurs par rapport au rendement professionnel de ces filles. D'où les questions suivantes.

Quelle image les populations de Kinshasa se font-elles du choix professionnel des filles déscolarisées? Dit autrement, comment réagissent-elles face à un réparateur de radios ou un mécanicien femme? Pourquoi ces filles ont-elles choisi d'exercer les métiers " masculins "? Quel est leur rendement? Quelle en est l'appréciation des employeurs ? Quels rapports entretiennent ces filles avec leurs collègues hommes?

Considérant que la donne culturelle exerce consciemment ou inconsciemment une influence déterminante sur les comportements de l'individu et ses jugements, la situation actuelle des femmes de Kinshasa engagées dans les métiers traditionnellement reconnus aux seuls hommes, peut constituer un choc culturel grave. Pour ce, nous avançons trois présupposés. Primo, en dépit du rendement satisfaisant que donneraient les filles dans l'exercice des métiers " masculins ", la population de Kinshasa en aurait une attitude ambivalente ; ainsi, les barrières culturelles et l'ignorance renforceraient les représentations négatives. Secundo, au-delà des raisons de survie créées par la situation de pauvreté, le choix par les filles déscolarisées d'apprendre et d'exercer les métiers « masculins » serait dû à leur estime personnelle et à la confiance en leurs capacités, renforcés par de nombreux forums internationaux sur l'émancipation de la femme et des actions des ONG locales incitant la femme à prendre courageusement de l'initiative dans tous les secteurs de la vie. Tertio, les filles exerçant les métiers « masculins » donneraient un rendement professionnellement satisfaisant, à l'instar de leurs collègues garçons. 
Les résultats de cette étude permettent de vérifier si les préjugés et le poids des valeurs culturelles traditionnelles continuent à faire obstacle à l'accès des femmes aux diverses opportunités économiques et aux métiers qui s'offrent à elles. Cela, même en milieu urbain comme la ville de Kinshasa et en plein vingt et unième siècle, où le discours sur l'égalité entre les sexes est plus retentissant. Concrètement la vérification de ces hypothèses permet d'atteindre trois objectifs : évaluer les attitudes des populations de Kinshasa face à l'apprentissage et l'exercice par les femmes des métiers " masculins "; identifier et analyser les motivations des filles déscolarisées, dans le choix desdits métiers; les observer en situation pour identifier la nature de rapports avec leurs collègues hommes ; et obtenir l'appréciation des employeurs sur leur rendement professionnel.

\section{Repères conceptuel et théorique sur l'image sociale des filles exerçant des métiers « masculins »}

Le concept d'" image sociale " que nous employons dans cet article traduit une forme de connaissance sociale, une pensée collective, socialement construite et partagée par les membres d'un même ensemble social ou culturel. Autrement qualifiée de représentation sociale, elle est déterminée à la fois par le système social et idéologique auquel appartiennent les individus et par les éléments idiosyncrasiques liés à l'histoire personnelle de chacun (Abric, I989; Doise et al. I992).

Dit autrement, ce concept traduit la manière dont les sujets sociaux appréhendent les événements de la vie courante et se portent à opérer les catégorisations, à attribuer aux individus et aux choses, les statuts, les rôles et les valeurs, selon les normes de références particulières à un moment donné. A cet égard, la division sexuelle de travail se fonde sur des considérations d'ordre culturel et subjectif, lesquelles conçoivent les métiers «masculins " comme ceux exigeant suffisamment d'énergie physique et intellectuel, tandis que les activités dites féminines semblent plus simples et peu sollicitant d'effort susmentionné. Cette connaissance socialement construite, n'est pas un fait statique. Elle naît, se développe et se déconstruit ; ce faisant elle détermine la nature de rapports entre les sujets ou groupes sociaux.

En terme d'analyse, nous convenons avec Fischer (I987: 4I) sur sa théorie, pour penser la représentation sociale comme " un processus d'élaboration perceptive et mentale de la réalité qui transforme les objets sociaux (personnes, contextes, situations) en catégories symboliques (valeurs, croyances, idéologies) et leur confere un statut cognitif, permettant d'appréhender les aspects de la vie ordinaire par un recadrage de nos propres conduites à l'intérieur des interactions sociales".

Appliquée à l'apprentissage et à l'exercice, par les femmes des métiers « masculins ", cette théorie nous permet de saisir et de comprendre la mentalité face aux rôles et compétences de la femme, dans une société marquée par des tabous. Aussi, en considérant particulièrement la recomposition de l'environnement international où, en plus du foisonnement des instruments juridiques et des discours sur la parité et l'égalité des sexes, il y a la montée remarquable d'un leadership féminin tant en Afrique qu'ailleurs, avec des femmes devenues chef d'Etat (au Liberia, au Malawi), chef de gouvernement (en Allemagne, au Sénégal), candidate aux élections à tous les niveaux, etc. 
Dans la mesure où ce modèle permet d'étudier la subjectivité propre aux représentations individuelles d'une population particulière (notamment celle de Kinshasa) sur le rôle de la femme, ses compétences et les types de métiers qu'elle doit exercer tels que présentés dans notre échelle d'attitude, nous avons utilisé cette théorie pour définir et expliquer la manière dont ladite population perçoit l'initiative actuelle de la femme d'exercer les métiers traditionnellement " masculins "; mais également pour savoir si les places occupées par les femmes et par les hommes, et les rôles sociaux que les unes et les autres jouent, sont simplement le résultat de la différence physiologique entre les sexes ou si elles sont plutôt le produit d'une longue construction collective.

Au delà de la représentation sociale, la motivation des filles au choix des métiers dits masculins est également analysée, comme un ensemble de causes, conscientes ou inconscientes, lesquelles permettent de mobiliser efficacement les efforts d'un individu ou groupe vers la réalisation de ses objectifs. Pour cette raison, un être humain peut préférer un objet à un autre, un domaine de formation à un autre, ou un type de métier à un autre.

De ce point de vue, la théorie de Porter et Lawler (I968) nous a été très utile, pour expliquer les raisons profondes qui portent des filles déscolarisées à franchir les barrières socialement construites, en exerçant les métiers dits " masculins ». Car, ces auteurs considèrent qu'un individu ne s'implique dans l'action que s'il s'estime capable de la réaliser et d'obtenir le résultat escompté, d'une part ; et s'il est assuré du profit qu'il en tirera et de la valeur qui sera accordée aux résultats, d'autre part.

\section{Implication méthodologique et échantillonnage}

En termes de choix méthodologique, les informations relatives aux filles apprenant les métiers masculins et l'image sociale qui en découle ont été obtenues au moyen de l'observation, du questionnaire et de l'échelle d'attitude. Organisées à l'aide de l'analyse de contenu, les données ont été traitées sur base des calculs des pourcentages. Mû par le souci de rendre opérationnelle la démarche de récolte et d'analyse des données, nous avons recouru à un échantillon raisonné de 250 sujets tirés de manière égale dans deux communes de Kinshasa (N'djili et Masina). Cet échantillon auquel le questionnaire et l'échelle d'attitude ont été soumis, compte I4o habitants des communes, 88 filles apprenantes de la mécanique et 22 formateurs.

Le répertoire des centres de métiers enregistrés au Ministère des Affaires Sociales a permis de sélectionner ces deux communes, en raison du grand nombre des centres de mécanique qu'elles comptent. Nous en avons tiré un échantillon de 22 centres dans lesquels nous avons travaillé avec l'ensemble des apprenantes et des formateurs, soit respectivement 88 filles et 22 formateurs. L'échantillonnage de ces centres a été fait au hasard au moyen de la technique de l'urne. Le choix de I40 habitants s'est fait de manière occasionnelle suivant leur disponibilité à répondre au questionnaire. Chacun des trois groupes composant l'échantillon est structuré suivant des caractères spécifiques, présentés ci-dessous. Concernant les habitants des communes, quatre indices ont été utilisés dans l'analyse de leurs représentations sociales, à savoir le sexe, le niveau d'étude, la tribu d'appartenance et le statut par rapport aux services des mécaniciens femmes. A ce 
effet, la répartition des sujets enquêtés se présente respectivement comme suit : 40,7\% sont des femmes et 59,3\% des hommes. Plus de la moitié des enquêtés (53,6\%) ont un niveau universitaire, $40,7 \%$ sont de niveau secondaire et 5,7\% de sujets sont du niveau primaire ; $63,6 \%$ des sujets enquêtés appartiennent aux tribus réputées intransigeantes ${ }^{3}$ à l'égard des initiatives de la femme, tandis que la proportion de ceux issus des tribus tolérantes ${ }^{4}$ est de $36,4 \%$; seuls $30 \%$ des personnes enquêtées ont déjà été dépannées par les mécaniciens femmes, tandis que $70 \%$ affirment n'avoir pas encore bénéficié de leurs services. Cette dernière catégorie serait composée des personnes ne disposant pas des véhicules. Elles sont vraisemblablement non intéressées par les services de dépannage de ces femmes. L'important ici est de déterminer leur attitude, par rapport aux services des mécaniciens femmes, en cas où ils disposaient d'un véhicule et que celui-ci tombait en panne. Concernant les filles apprenantes, deux variables ont été retenues pour analyser les réponses des filles enquêtées : type de famille de provenance et statut professionnel des parents. Les données recueillies indiquent que $58 \%$ des filles enquêtées proviennent des familles séparées, tandis que $42 \%$ sont issues des familles unies. Il en ressort que l'apprentissage de la mécanique intéresse tant les filles qui vivent dans une famille monoparentale que celles habitant avec les deux parents, bien que les filles de la seconde catégorie soient plus nombreuses ; 43,2 \% d'enquêtées avaient des parents sans emploi, $30,7 \%$ ceux exerçant un travail indépendant, tandis que seuls $26, \mathrm{I} \%$ d'enquêtées avaient des parents employés.

Au sujet des formateurs, seule la variable ancienneté a été retenue pour analyser leurs opinions. Il s'en suit que la majorité des enquêtés soit 59,I \% avaient une expérience supérieure à io ans dans le métier, 3I,8\% celle allant de 5 à Io ans, tandis que seuls 2 formateurs soit 9, I \% avaient l'ancienneté de 2 à 3 ans.

\section{Représentations sociales par rapport aux métiers masculins}

Il s'agit des images socialement construites par les habitants des communes enquêtées, concernant précisément la pertinence de la division sexuelle des métiers, le recours aux services d'un mécanicien femme, le fait d'avoir une fille ou une sœur mécanicienne, et la compatibilité entre les exigences de la mécanique et le statut d'épouse.

En République Démocratique du Congo, les coutumes de certaines tribus, à l'exemple des Baluba, sont généralement identifiées comme hostiles à l'épanouissement de la femme. Elles ne lui accordent pas beaucoup de considération et la placent le souvent au second rang. 
4.1. Pertinence de la division sexuelle des métiers

\begin{tabular}{l|c|c} 
Conceptions & Masculin & Féminin \\
\hline I. Les métiers sont indistincts des sexes & $\begin{array}{c}\% \\
\left(\mathrm{n}=\mathbf{8 3}_{3}\right.\end{array}$ & $\begin{array}{c}\% \\
(\mathrm{n}=57)\end{array}$ \\
\hline 2. Les métiers sont spécifiques à chaque sexe & 86,7 & 89,5 \\
\hline
\end{tabular}

Tableau 1 : Conceptions sur la division sexuelle des métiers, selon le sexe.

Il ressort de ce tableau que la conception selon laquelle les métiers sont indistincts de sexes est partagée par 89,5\% des femmes et 86,7\% d'hommes, contre Io,5 \% de femmes et $15,3 \%$ d'hommes qui se représentent les métiers comme étant naturellement spécifiques à chaque sexe. L'on constante qu'indépendamment de leurs sexes, les habitants des communes enquêtées considèrent dans leur écrasante majorité que la division sexuelle des métiers ne présente nullement de pertinence.

\begin{tabular}{l|c|c|c}
\multicolumn{1}{c|}{ Niveau d'études } & $\begin{array}{c}\% \\
(\mathrm{n}=8)\end{array}$ & $\begin{array}{c}\% \\
(\mathbf{n}=57)\end{array}$ & $\begin{array}{c}\% \\
(\mathbf{n}=75)\end{array}$ \\
\hline \hline Conceptions & 87,5 & 89,5 & 88 \\
\hline 2. Les métiers sont indistincts des sexes & Secondaire & Universitaire \\
\hline 2. Lers sont spécifiques à chaque sexe & $\mathrm{I} 2,5$ & $\mathrm{I} 0,5$ & $\mathrm{I} 2$
\end{tabular}

Tableau 2 : Conceptions sur la division sexuelle des métiers, selon le niveau d'étude.

Les données ci-dessus indiquent que la majorité des habitants des communes enquêtées, tout niveau d'études confondu, conçoivent les métiers comme étant indistincts des sexes. Cette conception est partagée par $87,5 \%$ des sujets du niveau primaire, $89,5 \%$ du niveau secondaire et $88 \%$ des sujets du niveau universitaire. La tendance contraire, qui considère la distinction sexuelle des métiers comme une réalité naturelle et typique à chaque sexe, est mentionnée par seulement I sujet du niveau primaire (soit I2,5\%), 6 sujets du niveau secondaire (soit I0,5\%) et 9 sujets du niveau universitaire (soit I2 \%). 


\begin{tabular}{l|c|c}
\multicolumn{1}{c|}{ Attitude de la tribu d'origine } & $\begin{array}{c}\text { Intransigeance } \\
(\mathbf{n = 8 9})\end{array}$ & $\begin{array}{c}\text { Tolérance } \\
(\mathbf{n = 5})\end{array}$ \\
\hline Conceptions & 89,9 & 84,3 \\
\hline I. Les métiers sont indistincts des sexes & I0,I & I5,7
\end{tabular}

Tableau 3 : Conceptions sur la division sexuelle des métiers, selon l'attitude de la tribu d'appartenance vis à vis de la femme et de ses initiatives.

Il ressort de ce tableau qu'indépendamment de la tribu d'origine, la majorité des habitants des communes enquêtées s'opposent à la conception qui prône la division sexuelle des métiers. En effet, $89,9 \%$ des enquêtés provenant des tribus aux coutumes jugées intransigeantes vis-à-vis de la femme et $84,3 \%$ de ceux issus des tribus marquées par une certaine tolérance pensent que les métiers peuvent être indistinctement exercés par les hommes et les femmes. Par contre, seule une minorité comprenant Io,I \% des enquêtés de la première catégorie et $15,7 \%$ de ceux de la seconde catégorie se représente la distinction sexuelle des métiers comme une donne naturelle et utile.

\begin{tabular}{|c|c|c|}
\hline & Bénéficiaire & Non Bénéficiaire \\
\hline Conceptions $\quad$ Statut & $\begin{array}{c}\% \\
(n=42)\end{array}$ & $\begin{array}{c}\% \\
(n=98)\end{array}$ \\
\hline I. Les métiers sont indistincts des sexes & 85,7 & 88,8 \\
\hline 2. Les métiers sont spécifiques à chaque sexe & $\mathrm{I} 4,3$ & II, 2 \\
\hline
\end{tabular}

Tableau 4 : Conceptions des enquêtés, selon le statut de bénéficiaire ou non des services des mécaniciens femmes.

Considérant le statut des enquêtés, selon qu'ils sont bénéficiaires ou non des services des mécaniciens femmes, ce tableau indiquent dans leur grande majorité $(85,7 \%$ des sujets bénéficiaires des services des femmes mécaniciennes et $88,8 \%$ des non bénéfciaires), les enquêtés conçoivent les métiers comme étant indistincts de sexes. Par contre, seulement I $4,3 \%$ des bénéficiaires desdits services et II, $2 \%$ des non bénéficiaires pensent que les métiers sont typiques à chaque sexe. 


\begin{tabular}{|c|c|c|}
\hline \multirow[t]{2}{*}{ Variables } & \multirow[t]{2}{*}{ Opinions } & Favorable \\
\hline & & $\begin{array}{c}\% \\
(n=I 40)\end{array}$ \\
\hline I. Sexe & $\begin{array}{l}\text { I. Masculin } \\
\text { 2. Féminin }\end{array}$ & $\begin{array}{l}59,3 \\
49,7\end{array}$ \\
\hline II. Niveau d'études & $\begin{array}{l}\text { I. Primaire } \\
\text { 2. Secondaire } \\
\text { 3. Universitaire }\end{array}$ & $\begin{array}{c}5,7 \\
40,7 \\
53,6\end{array}$ \\
\hline III. Attitudes de la tribu d'appartenance & $\begin{array}{l}\text { I. Intransigeance } \\
\text { 2. Tolérance }\end{array}$ & $\begin{array}{l}63,6 \\
36,4\end{array}$ \\
\hline IV. Statut & $\begin{array}{l}\text { I. Bénéficiaire } \\
\text { 2. Non bénéficiaire }\end{array}$ & $\begin{array}{l}30 \\
70\end{array}$ \\
\hline
\end{tabular}

Tableau 5 : Opinions des enquêtés sur le recours aux services d'un mécanicien femme, selon le sexe, le niveau d'étude, l'attitude de la tribu d'appartenance et le statut.

Le tableau ci-dessus donne une vue globale des opinions des I4o habitants des communes enquêtées concernant leur choix d'utiliser les services des mécaniciens femmes. Il en ressort une unanimité effective quant à l'acceptation des enquêtés d'être dépanné par lesdites femmes. Dit autrement, indépendamment du sexe, du niveau d'études, de l'attitude de la tribu d'appartenance et du statut de bénéficiaires des services ou non, les habitants des communes enquêtées s'estiment disposés à utiliser les services offerts par des mécaniciens femmes et d'être dépannés par ces dernières.

4.3. Avoir une fille ou une sœur mécanicienne

\begin{tabular}{l|c} 
Effectifs & $\begin{array}{c}\% \\
\text { (n=I40) }\end{array}$ \\
\hline I. Oui, j'accepterai, si ma fille/sœur choisit d'apprendre la mécanique & 87,9 \\
\hline 2. Non, je n'accepterai pas & I2,I
\end{tabular}

Tableau 6: Opinions sur le choix d’avoir une fille/søurs mécanicienne.

Les données de ce tableau permettent d'appréhender les sentiments qu'éprouvent les habitants des communes enquêtées, face à leurs filles ou sœurs qui s'intéresseraient au métier de mécanicien. A la question de savoir s'ils peuvent permettre à leurs filles ou sœurs d'apprendre ou d'exercer la mécanique, la grande majorité des enquêtés, I23 personnes soit $87,9 \%$ se disent favorables et n'en trouvent aucun inconvénient, contre 17 autres soit I2,I \% qui affichent un refus catégorique. Ce choix différentiel trouve son fon- 
dement dans la conception sur la division sexuelle des métiers, présentée aux tableaux 5, 6, 7 et 8. En effet, les enquêtés qui acceptent que leurs filles/sœurs fassent la mécanique, sont les mêmes qui ont une représentation positive des métiers, les considérant indistincts de sexes. Par contre, ceux qui s'y opposent sont les enquêtes qui pensent que la mécanique est un métier spécifiquement masculin, et donc, non adapté aux femmes.

4.4. Compatibilité entre les exigences de la mécanique et le statut d'épouse

\begin{tabular}{|c|c|}
\hline Effectifs & $\begin{array}{c}\% \\
(n=I 40)\end{array}$ \\
\hline I. Les devoirs du mécanicien sont compatibles avec le statut d'épouse & $87, \mathrm{I}$ \\
\hline $\begin{array}{l}\text { 2. Les exigences de la mécanique ne sont pas compatibles avec le sta- } \\
\text { tut d'épouse }\end{array}$ & 12,9 \\
\hline
\end{tabular}

Tableau 7 : Opinions des habitants des de Kinshasa sur la compatibilité entre les exigences de la mécanique et le statut d'épouse.

Les informations du tableau II exposent les opinions des enquêtés concernant la compatibilité entre les exigences de la mécanique et le statut d'épouse. La majorité des enquêtés, soit $87, \mathrm{I} \%$, estiment que le métier de mécanique ne peut nullement empêcher à une femme d'accomplir ses devoirs ménagers. A l'instar des autres métiers qu'exercerait une femme, la mécanique lui exige l'effort, la disponibilité, l'engagement et le dévouement. Alors que pour I2 \% des enquêtés, le travail au garage est très absorbant et physiquement épuisant pour une femme.

4.5. Motivations aux métiers masculins

\begin{tabular}{l|c|c|c|c}
\hline \multirow{2}{*}{ Motivations } & $\begin{array}{c}\text { Sans } \\
\text { emploi }\end{array}$ & Indépendant & Employé & Total \\
\cline { 2 - 5 } & $\begin{array}{c}\% \\
(\mathbf{n = 3 8 )}\end{array}$ & $\begin{array}{c}\% \\
(\mathbf{n = 2 7})\end{array}$ & $\begin{array}{c}\% \\
(\mathbf{n = 2 3})\end{array}$ & $\begin{array}{c}\% \\
(\mathbf{n}=\mathbf{8 8})\end{array}$ \\
\hline I. Amour du métier & I00 & I00 & I00 & I00 \\
\hline 2. Assurance d'en posséder les aptitudes & 97,4 & 96,3 & 95,7 & 96,6 \\
\hline 3. Gagner de l'argent & $\mathrm{I3,2}$ & 7,4 & $\mathrm{I7,4}$ & I2,5 \\
\hline 4. Exemples tirés d'autres femmes & I00 & I00 & I00 & I00
\end{tabular}

Tableau 8: Motivations à l’apprentissage de la mécanique, selon le statut professionnel des parents.

Il ressort des données ci-dessus qu'indépendamment du statut professionnel des parents, la motivation des enquêtées est fonction de l'amour du métier, de l'assurance d'en posséder les aptitudes, du bénéfice financier à gagner et des exemples tirés d'autres 
femmes exerçant la mécanique. Les taux des réponses relatives à ces déterminants sont respectivement de $100 \%, 97,4 \%, \mathrm{I}_{3}, 2 \%$ et $100 \%$ chez les filles dont les parents sont sans emploi ; 100 \%, 96,3 \%, 7,4 \% et roo \% chez celles dont les parents exercent un travail indépendant ; I00 \%, 95,7\%, 17,4 \% et I00 \% chez les enquêtées dont les parents sont des employés.

Au regard de ces chiffres, l'amour du métier et l'assurance d'en posséder les aptitudes sont les principales raisons ayant poussé à l'apprentissage de la mécanique, suivi largement du modèle social tiré des femmes exerçant des métiers semblables. Loin d'être une simple activité pour la survie, l'apprentissage de la mécanique résulte d'un choix rationnel des filles conscientes de leurs capacités à travailler concurremment avec les hommes, ayant surmonté le complexe lié au genre.

\begin{tabular}{|c|c|c|c|}
\hline \multirow[b]{2}{*}{ Motivations } & $\begin{array}{l}\text { Famille } \\
\text { unie }\end{array}$ & $\begin{array}{l}\text { Famille } \\
\text { séparée }\end{array}$ & Total \\
\hline & $\begin{array}{c}\% \\
(n=37)\end{array}$ & $\begin{array}{c}\% \\
(n=51)\end{array}$ & $\begin{array}{c}\% \\
(n=88)\end{array}$ \\
\hline I. Amour du métier & IOO & IOO & 100 \\
\hline 2. Assurance d'en posséder les aptitudes & 94,6 & 98,03 & 96,6 \\
\hline 3. Gagner de l'argent & Io, 8 & $\mathrm{I} 3,7$ & $\mathrm{I} 2,5$ \\
\hline 4. Exemples tirés d'autres femmes & IOo & IOo & IOO \\
\hline
\end{tabular}

Tableau 9: Motivations à l'apprentissage de la mécanique, selon le type de familles d'origine.

Considérées selon le type des familles d'origine, la tendance des réponses fournies par ce tableau demeure la même que celle du tableau I2. Les taux des réponses relatives aux quatre motivations à la mécanique sont respectivement de 100 \%, 94,6\%, I0,8\% et I00 \% chez les filles provenant des familles unies ; tandis que pour celles issues des familles séparées, ces taux sont respectivement de ı०o \%, 98,03\%, I3,7 \% et I00 \%. En hiérarchisant les différentes motivations, on remarque, comme pour le statut professionnel des parents, qu'indépendamment des familles d'origine, les enquêtées sont unanimement portées par l'amour de la mécanique et le modèle social tiré des femmes exerçant des métiers semblables, puis majoritairement par l'assurance de posséder les capacités à exercer la mécanique. Enfin, par le gain financier à obtenir.

\subsection{Niveau de performance des filles mécaniciennes}

L'évaluation des performances des femmes mécaniciennes, faite respectivement par leurs formateurs et leurs collègues hommes, est présentée dans les tableaux ci-dessous. 


\begin{tabular}{|c|c|c|c|c|}
\hline Ancienneté des formateurs & 2 à zans & 5 à ro ans & Plus de ro ans & Total \\
\hline Niveau de performance & $\begin{array}{c}\% \\
(n=2)\end{array}$ & $\begin{array}{c}\% \\
(\mathrm{n}=7)\end{array}$ & $\begin{array}{c}\% \\
(\mathrm{n}=\mathrm{I} 3)\end{array}$ & $\begin{array}{c}\% \\
(\mathrm{n}=22)\end{array}$ \\
\hline $\begin{array}{l}\text { I. Excellent, supérieur à celui des } \\
\text { garçons }\end{array}$ & - & - & - & - \\
\hline $\begin{array}{l}\text { 2. Très bon, comparable à celui des } \\
\text { garçons }\end{array}$ & $9, \mathrm{I}$ & $3 \mathrm{I}, 8$ & $59, \mathrm{I}$ & IOO \\
\hline 3. Faible, inférieur à celui des garçons & - & - & - & - \\
\hline
\end{tabular}

Tableau 10 : Appréciation de la performance des filles mécaniciennes, selon l'ancienneté du formateur.

Ce tableau renseigne sur le niveau de performance des filles apprenant ou exerçant la mécanique. Il s'agit de l'appréciation donnée par des formateurs qui sont aussi leurs patrons, laquelle est basée sur le savoir et le savoir-faire professionnel des mécaniciens femmes. A l'unanimité, tous les formateurs interrogés, soit Ioo \%, reconnaissent aux filles évoluant dans leurs garages un très bon niveau de performance comparable à celui de leurs collègues garçons.

\begin{tabular}{|c|c|}
\hline Opinions des mécaniciens hommes & $\begin{array}{c}\% \\
(n=24)\end{array}$ \\
\hline I. Les filles manifestent des compétences remarquables en mécanique & $9 \mathrm{I}, 7$ \\
\hline 2. Les filles éprouvent d'énormes difficultés en mécanique & 8,3 \\
\hline
\end{tabular}

Tableau 11 : Opinions des mécaniciens hommes sur les compétences de leurs collègues femmes

Interrogés sur les compétences de leurs collègues femmes, les mécaniciens hommes ont émis des avis non concordants mais avec prédominance d'opinions favorables. En effet, alors que seuls 8,3 \% des enquêtés doutent des compétences des femmes, en relevant d'énormes difficultés qu'elles éprouvent dans le métier, la majorité, soit 9I,7 \% d'enquêtés, assure que les femmes manifestent des compétences remarquables en mécanique. Cette appréciation corrobore tant les avis des populations sur l'efficacité des mécaniciens femmes (voir tableau 9) que l'appréciation majoritairement donnée par leurs formateurs/ patrons quant à leur niveau de performance (tableau Io).

\section{Décryptage des résultats et retour aux questions de départ}

5.1. Concernant l'attitude ambivalente des populations de Kinshasa face aux filles exerçant les métiers " masculins"

La grande majorité des enquêtés (88 \%) s'oppose à la division sexuelle des métiers, laquelle supposerait une distribution inégale des aptitudes à la naissance, entre homme et femme. En dépit de leur sexe, de la différence du niveau d'études, des bénéfices tirés 
des services des filles mécaniciennes ou non, et malgré leur appartenance aux tribus marquées par une intransigeance vis à vis de la femme et de ses initiatives, nombre d'enquêtés n'admettent pas la conception qui prône la distinction sexuelle des métiers, estimant qu'il n'en existe pas qui soient typiquement masculins ou féminins. Selon les compétences requises, les métiers peuvent être indistinctement exercés par l'homme et la femme, chacun porté à l'activité qui l'intéresse.

L'attitude positive ainsi majoritairement observée reflète une évolution par rapport aux conceptions culturelles, établissant les frontières entre les métiers masculins et féminins. Elle traduit probablement le bénéfice acquis du contexte national actuel sur les tabous culturels ; contexte marqué par d'abondantes actions de sensibilisation contre la discrimination de la femme. La minorité qui soutient l'existence des métiers pour homme et pour femme resterait encore subjuguée par la coutume et par des convenances personnelles. Elle serait aussi éventuellement ignorante du débat contemporain sur la question du genre, ainsi que des instruments juridiques nationaux et internationaux à ce sujet.

En dépit de cela, tous les habitants des communes enquêtés acceptent unanimement d'utiliser les services des mécaniciens femmes, en cas de besoins. Cette unanimité implique que l'image socialement construite sur les femmes exerçant les métiers « masculins » repose sur des valeurs objectives, tels le savoir-faire et la compétence, plutôt que simplement sur des considérations d'ordre culturel. Les preuves sont ainsi données en considérant, tant le statut des bénéficiaires que la tribu de provenance. Pour cela, bien que $6_{3}, 6 \%$ des enquêtés soient issus des tribus réputées intransigeantes à l'égard de la femme et que $70 \%$ n'aient pas encore bénéficié des services de mécaniciens femmes, les enquêtés sont entièrement favorables au choix et à la pratique professionnels de celles-ci. Même la minorité qui a considéré la division sexuelle de travail comme un fait naturel, affirme pouvoir recourir, si besoin est, aux services des mécaniciens femmes. S'agiraitil d'un choix par défaut, lequel serait dicté par la nécessité, faute de mieux ? Qu'à cela ne tienne, une réalité demeure : accepter les services des mécaniciens femmes, même si c'est par nécessité, traduit une reconnaissance de leur capacité professionnelle.

Ces appréciations résultent des jugements personnels opérés par les uns et les autres, en fonction des valeurs auxquelles ils adhèrent. Dans la ville de Kinshasa où il n'y a pas une tribu dominante qui impose sa culture, les habitudes et les comportements des habitants sont influencés par les valeurs découlant du choc des cultures et du discours contre toutes les formes de ségrégation liées au genre. L'adhésion à ce discours progressiste et les preuves apportées des compétences manifestées par des femmes dans divers domaines ont produit des nouvelles représentations sur la femme et ses initiatives éventuelles. Indépendamment du sexe, du niveau d'études, de l'attitude de la tribu d'appartenance et du statut de bénéficiaires des services, 87,9 \% d'habitants des communes où l'enquête a eu lieu enquêtés affirment pouvoir respecter, voire encourager le choix de leurs filles ou sœurs, si ces dernières optent pour le métier de mécanicien. En effet l'engagement d'un apprenant dans un processus d'apprentissage dépend foncièrement de l'intérêt qu'il en tire. Pour cela, chaque personne doit jouir de la liberté 
de choisir le type de formation qui l'intéresse et corresponde à ses capacités, le rendement à réaliser en dépendant forcément.

Ces résultats infirment la première hypothèse avancée dans cette étude selon laquelle, la population de Kinshasa aurait une attitude ambivalente face à l'exercice des métiers « masculins " par les filles. Contrairement à cette supposition, les habitants des communes enquêtées s'affichent très favorables à l'apprentissage et à l'exercice par les filles des métiers dits masculins.

Il est donc aberrant de préjuger de l'incapacité des femmes à s'adapter aux « métiers masculins ", telle que la mécanique, dont les exigences seraient incompatibles tant au statut d'épouse qu'aux devoirs ménagers ; ou de prétendre que l'effort physique exigé par ces métiers déformerait la morphologie de la femme, la rendant plus musclée et moins jolie, et réduirait ainsi ses chances pour le mariage.

A l'instar des autres métiers qu'exercerait une femme, la mécanique requiert l'effort, la disponibilité, l'engagement et le dévouement. Le mécanicien femme devra, comme pour les autres métiers, savoir organiser son temps pour que son travail au garage n'empiète pas sur ses tâches domestiques. Ces exigences sont aussi valables pour la femme médecin, infirmière, comptable, enseignante, etc. Si nombre de femmes mariées exerçant les métiers précités, réussissent à les combiner avec les tâches ménagères, celles exerçant la mécanique le feraient pareillement, toute choses restant égales par ailleurs.

\subsection{A propos des motivations des filles aux "métiers masculins"}

Le choix de la mécanique par les filles déscolarisées se fonde sur leur conception du travail, la valeur qu'elles y accordent, l'auto-perception de leurs capacités et le modèle social tirés d'autres femmes exerçant les métiers semblables, dont elles se sont inspirées. A l'unanimité, toutes les enquêtées affirment avoir rencontré plusieurs femmes exerçant les activités autrefois pratiquées par seulement les hommes, à l'exemple de chauffeurs, laveuses des voitures, photographes, boulangères, militaires, etc. Convaincues qu'il n'y a pas de sot métier, les filles n'hésitent pas à s'engager dans les activités professionnelles qui assurément leur procurent un revenu. Mais, bien plus dans celles où elles s'estiment capables.

Ces résultats confirment notre deuxième hypothèse qui stipule qu'au delà des raisons de survie créées par la situation de pauvreté, le choix par les filles déscolarisées d'apprendre et d'exercer les métiers « masculins " serait dû à leur estime personnelle et à la confiance en leurs capacités.

Mieux s'appliquer dans un travail requiert la volonté et la possession des aptitudes (savoir et savoir-faire), lesquelles ne se développent que par l'exercice. L'amour de la mécanique et la prise de conscience des aptitudes à l'exercer, ont stimulé les filles à intégrer différents garages pour apprendre. Pour cela, affirme Musa Alokpo (2009), l'intérêt pour ce qu'on apprend est un déterminant essentiel pour pouvoir y focaliser son attention et offrir sa participation. La pédagogie active recommande que l'enseignement soit attrayant et qu'il réponde aux attentes des apprenants. De même, les études en psychologie de la personne adulte reconnaissent que ce dernier ne s'engage en formation qu'en fonction des réponses qu'il attend obtenir à ses besoins et intérêts (Roussel, I996; Bandura, I986). 
Ainsi, contrairement à ce qui est généralement avancé, le contexte de pauvreté ne constitue pas la seule explication de la motivation des filles aux métiers " masculins ". Ces filles, visent bien sûr un gain financier partant de leur travail ; mais, elles ont surtout une auto-appréciation positive de leurs aptitudes, s'estimant capable d'exercer concurremment avec les garçons, les mêmes métiers et d'obtenir les résultats escomptés. Ces différents éléments constituent, selon Porter et Lawle, les facteurs déterminants de la motivation.

Le monde est actuellement en pleine évolution; le marché du travail ne doit donc pas rester en marge des changements qui s'y opèrent. Partout au monde, les femmes se sont insérées dans presque tous les secteurs socio-professionnels, où elles réalisent des performances qualitativement comparables voire supérieures à celles de leurs collègues hommes. Raison pour laquelle plusieurs de celles qui sont qualifiées occupent des postes de commandement dans le secteur formel du travail. Ces faits démontrent donc l'erreur qu'il y a de présumer de la possession des aptitudes par rapport au sexe des individus. C'est dans cette perspective que les organisations non gouvernementales locales, s'appuyant sur les instruments juridiques internationaux, ont intensifié les activités de sensibilisation en faveur de la parité, l'égalité et l'épanouissement de la femme. Cette sensibilisation conscientisante a créé une grande motivation dans l'engagement professionnel des filles dans divers métiers.

\subsection{Concernant les compétences des filles dans les "métiers masculins"}

Les opinions émises par les formateurs/patrons et les mécaniciens hommes concernant le savoir-faire professionnel des femmes exerçant la mécanique sont convergentes. Ces femmes font preuve des compétences remarquables. Certes, la réalisation des travaux comme le transport et le soulèvement de certains matériaux, le serrage et le desserrage des boulons, le découpage des métaux ou autres, exige beaucoup d'effort physique pour lequel les hommes semblent plus aptes que les femmes. Cependant, le recours à la technologie moderne et à l'outillage approprié permet aux mécaniciens femmes de réaliser d'excellentes performances pareillement à leurs collègues hommes.

Ces résultats confirment la troisième hypothèse selon laquelle les filles exerçant les métiers «masculins » donneraient un rendement professionnellement satisfaisant, à l'instar de leurs collègues garçons.

\section{Conclusion}

Contrairement à ce qu'on aurait pensé, les habitants de Kinshasa (enquêtés) ont été, dans leur grande majorité, favorables à l'exercice des métiers " masculins " par les femmes. L'apprentissage de la mécanique par les filles déscolarisées est perçu comme un début de progrès par rapport aux coutumes, lesquelles séparent le champ d'activités de l'homme de celui de la femme. Les populations concernées par cette étude, indépendamment des variables considérées, se montrent favorables à la désexualisation des métiers, 
pour stimuler la participation des femmes aux diverses professions et activités de production. En référence à la théorie de Fischer G., l'on note qu'il y a une grande évolution dans la conception de la division de travail par rapport au sexe. Les considérations d'ordre culturel et subjectif se déconstruisent de plus en plus, en faveur d'une conception plus objective, reconnaissant la possession des aptitudes, indistinctement à l'homme et à la femme. Cette image positive de la femme et de ses capacités influe inéluctablement sur son statut et rapports sociaux.

Dans les garages où elles apprennent la mécanique, les filles font preuve des compétences remarquables, à l'instar de leurs collègues garçons ; et cela, à la grande satisfaction de leurs patrons. Incitées par les organisations non gouvernementales (féministes) locales, les filles déscolarisées ont pris conscience du fait que le travail ennoblit l'être humain et le libère de l'oisiveté et de la dépendance. De même, estiment-elles, l'aptitude à réaliser un travail n'est pas une donne sexuelle ; elle est donnée aux hommes et aux femmes. Cette prise de conscience les a portées à franchir les barrières culturelles ou idéologiques relatives aux métiers " masculins " et " féminins ".

Ainsi, autant il est nécessaire de créer des structures d'apprentissage des métiers pour encadrer les filles déscolarisées (lesquelles deviennent de plus en plus nombreuses), autant il devient impérieux que soient continuellement menées des campagnes de sensibilisation et de conscientisation, pour inviter certaines populations qui demeurent encore sous les pesanteurs culturelles ou religieuses à changer de mentalité et à bannir les inégalités entre hommes et femmes dans l'accès au marché de travail et l'exercice des différents métiers.

\section{Références}

Abric, J.-C. (1989). L'étude expérimentale des représentations sociales. In D. Jodelet (dir.), Les représentations sociales. pp. I87-204. Paris: Presses Universitaires de France.

Bandura, A. (1986). Social foundations of thought and action : a Social Cognitive Theory. Prentice-Hall : Englewood Cliffs, $544 \mathrm{p}$.

Berner, B. (I997). L'ingénierie ou le génie du male : masculinité et enseignement technique au tournant du $20 e$ siècle. Travail, espaces et professions in cahiers de Gedisst, $n^{\circ}$ I9. pp. 7-25. Paris, l'Harmattan.

Chataigner, J-M et Magro, H. (2007). Etats et sociétés fragiles : entre conflit, reconstruction et développement. Paris, Karthala, $565 \mathrm{p}$.

Convention sur l'Elimination de toutes les formes de discrimination à l'égard de la femme 1979, (http://www.unhchr.ch/french/html/menu3/b/ercedaw_fr.htm). Consulté le 3 Septembre 2013.

Déclaration universelle des droits de l'homme du ro décembre 1948.

Doise, W., Clémence, A. et Lorenzi-Cioldi, W. (1992). Représentations sociales et analyse de données. Grenoble: Presses Universitaires de Grenoble, 264 p.

Duruflé, G. (I988). L'ajustement structurel en Afrique (Sénégal, Côte d'Ivoire, Madagascar). Paris : Karthala, 205 p.

Etounga-Manguelle D. (I994). De l'ajustement structurel à l'ajustement culturel: la seule solution pour l'Afrique (http://www.euforic.org), Consulté le I3 novembre 2013,

Fischer, G. (1987). Les concepts fondamentaux de la psychologie sociale. Montréal : Dunod, 278 p.

Lachaud, P. (1997). Les femmes et le marchédu travail urbain en Afrique subsaharienne. Paris : L’Harmattan, $233 \mathrm{p}$.

Massiah, G. (2000). Grandeur et décadence de l'ajustement structurel (http://www.globenet.org/aitec/contributions), consulté le ro Février 2013. 
Musa Alokpo, D. (2009). Analyse de l'efficacité de l'éducation non formelle dans la ville de Kinshasa. Une contribution à la politique de l'éducation, Thèse de doctorat inédite, Kinshasa, Université de Kinshasa, 4II p.

Porter, L. W. et Lawler, E. E. (I968). Managerial attitudes and performance. Homewood, III : Dorsey Press and Richard D. Irwin, $209 \mathrm{p}$.

Roussel, P. (1996). Rémunération, motivation et satisfaction au travail. Paris : Editions Economica,306 p.

Trefon, T. (2004). La réinvention de l'ordre à Kinshasa. In Cahiers Africains, n6I-62, séries 2003, pp I5-3I.

Séhili, D. (2003). La castration sociale. Paris : Syllepse, 200 p.

Simkins, T. (1977). La planification de l'éducation extrascolaire : stratégies et contraintes. In perspectives, Vol. VIII, n 2, pp. 20I-2I2, Paris: Unesco. 